Prepared in cooperation with the Wildlife Disease Association Workshop held in conjunction with the 2016 Annual Wildlife Disease Association Conference, Greek Peak Mountain Resort, New York

\title{
Report of the Workshop on Evidence-Based Design of National Wildlife Health Programs
}
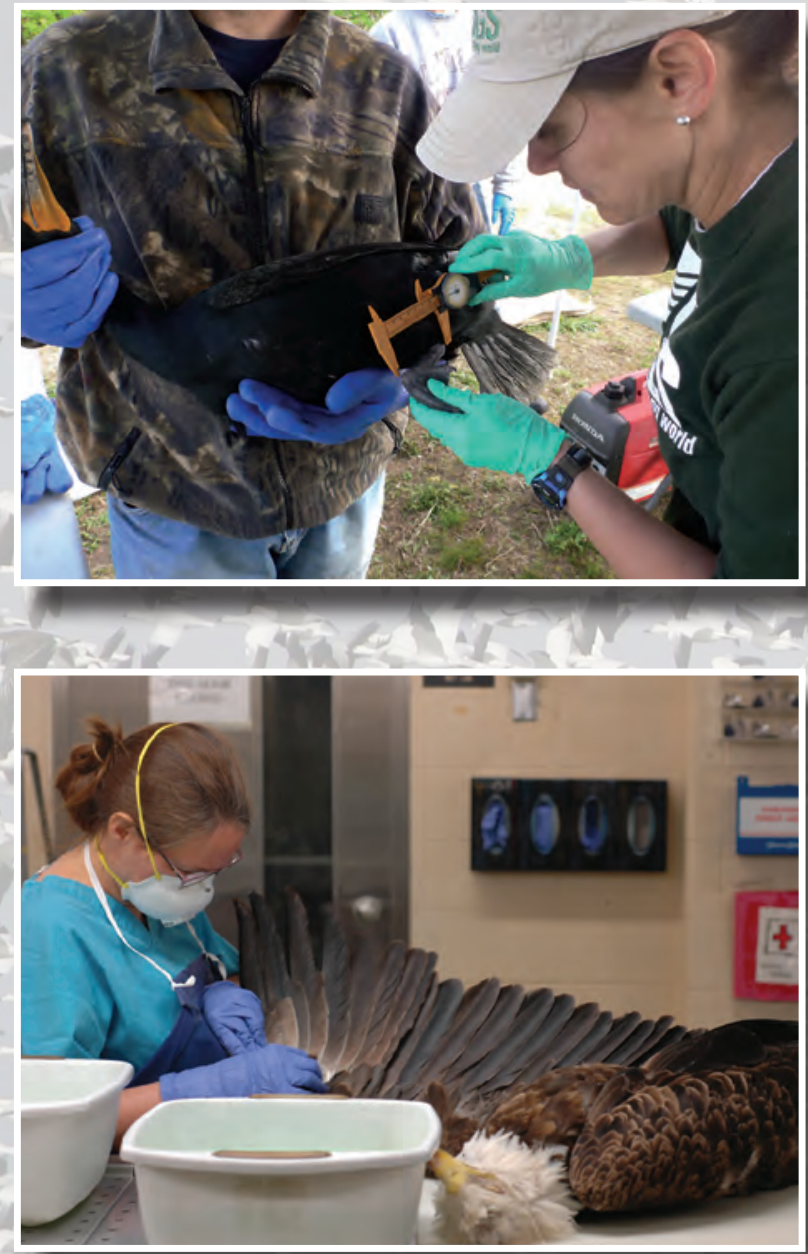

Open-File Report 2017-1038
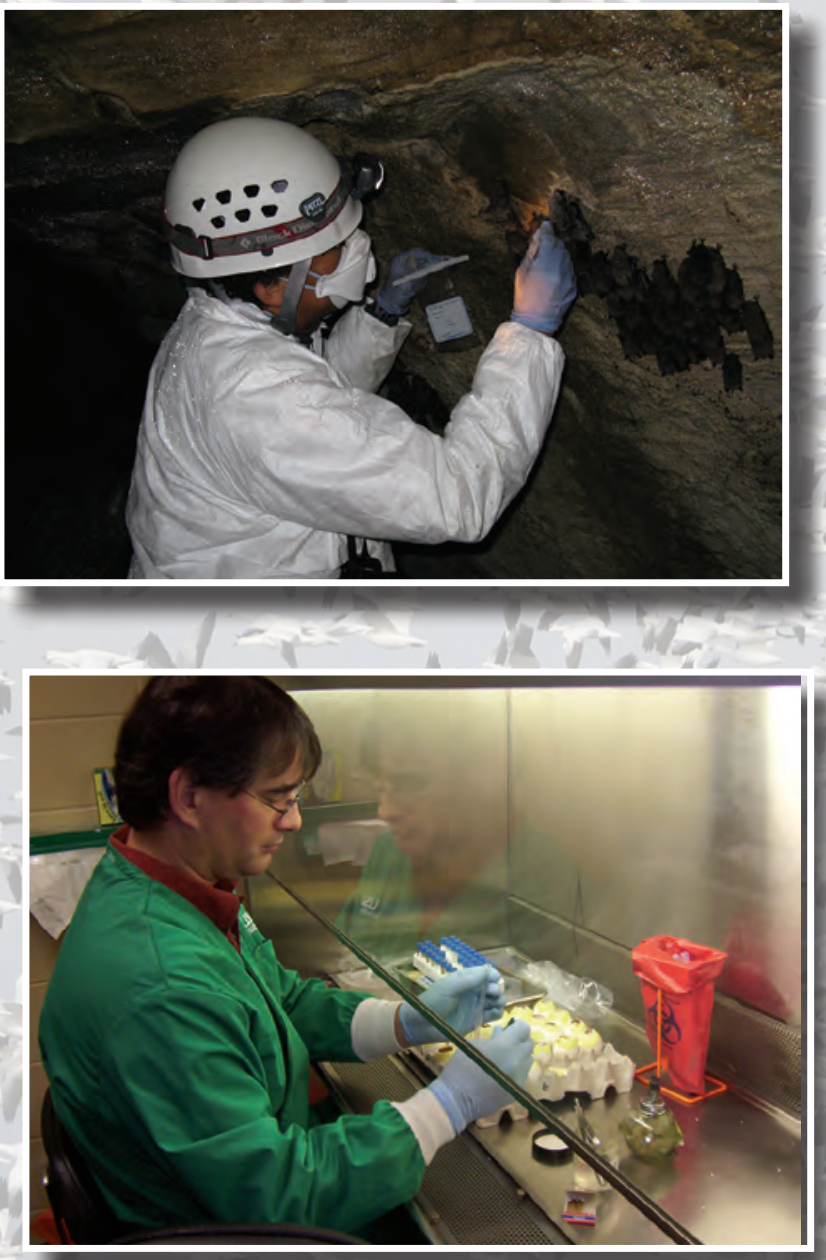

U.S. Department of the Interior

U.S. Geological Survey

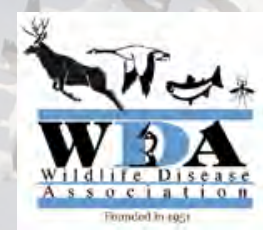


Cover images, clockwise, starting top left:

Top left, USGS scientist measures the tarsus length of a double-crested cormorant for a study on Newcastle disease virus (USGS photo).

Top right, USGS scientist taking samples from a cave for a study on white-nose syndrome in bats (USGS photo).

Bottom right, USGS biological science technician testing samples for avian influenza virus by inoculating embryonating eggs (USGS photo).

Bottom left, USGS scientist inspecting wing of a bald eagle that will be tested for lead poisoning (USGS photo).

Background photo of snow geese (Photo by Bob Dusek). 


\section{Report of the Workshop on Evidence-Based Design of National Wildlife Health Programs}

By Natalie T. Nguyen, J. Paul Duff, Dolores Gavier-Widén, Tiggy Grillo, Hongxuan He, Hang Lee, Parntep Ratanakorn, Jolianne M. Rijks, Marie-Pierre Ryser-Degiorgis, Jonathan M. Sleeman, Craig Stephen, Toni Tana, Marcela Uhart, and Patrick Zimmer (authors are listed in alphabetical order after first author)

Prepared in cooperation with the Wildlife Disease Association Workshop held in conjunction with the 2016 Annual Wildlife Disease Association Conference, Greek Peak Mountain Resort, New York

Open-File Report 2017-1038 


\title{
U.S. Department of the Interior \\ RYAN K. ZINKE, Secretary
}

\section{U.S. Geological Survey William H. Werkheiser, Acting Director}

\author{
U.S. Geological Survey, Reston, Virginia: 2017
}

For more information on the USGS - the Federal source for science about the Earth, its natural and living resources, natural hazards, and the environment-visit https://www.usgs.gov or call 1-888-ASK-USGS.

For an overview of USGS information products, including maps, imagery, and publications, visit https://store.usgs.gov.

Any use of trade, firm, or product names is for descriptive purposes only and does not imply endorsement by the U.S. Government.

Although this information product, for the most part, is in the public domain, it also may contain copyrighted materials as noted in the text. Permission to reproduce copyrighted items must be secured from the copyright owner.

Suggested citation:

Nguyen, N.T., Duff, J.P., Gavier-Widén, D., Grillo, T., He, H., Lee, H., Ratanakorn, P., Rijks, J.M., Ryser-Degiorgis, M.-P., Sleeman, J.M., Stephen, C., Tana, T., Uhart, M., and Zimmer, P., 2017, Report of the workshop on evidence-based design of national wildlife health programs: U.S. Geological Survey Open-File Report 2017-1038, 18 p., https://doi. org/10.3133/ofr20171038.

ISSN 2331-1258 (online) 


\section{Acknowledgments}

This workshop was supported by the Wildlife Disease Association Small Grants Program, the U.S. Geological Survey, and the Canadian Wildlife Health Cooperative. The participants also acknowledge the Division of Wildlife Diseases, Swedish Veterinary Authority; the Department for Environment, Food and Rural Affairs, the Animal and Plant Health Agency - Wildlife Expert Group, and the Great Britain Wildlife Disease Surveillance Partnership; the New Zealand Ministry for Primary Industries; and the Australian Government Department of Agriculture and Water Resources. We thank Rupert Woods (Wildlife Health Australia) for his input and support, and Drs. Julie Lenoch, Iga Stasiak, and Michelle Verant for helpful comments on this report. 



\section{Contents}

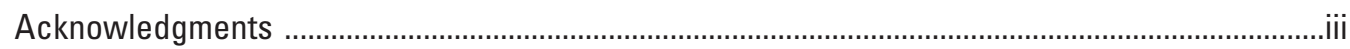

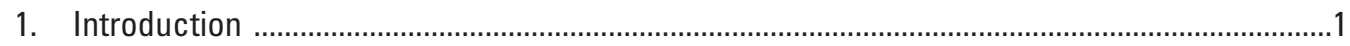

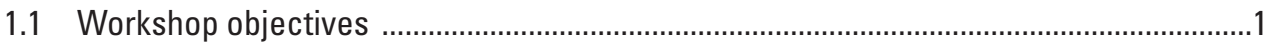

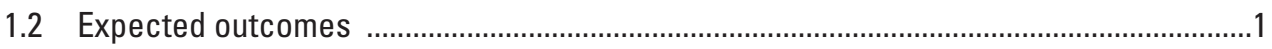

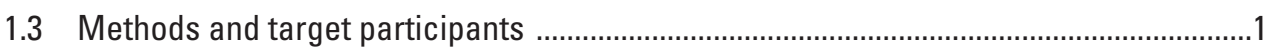

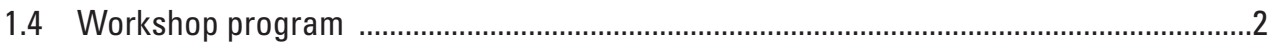

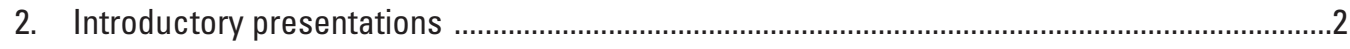

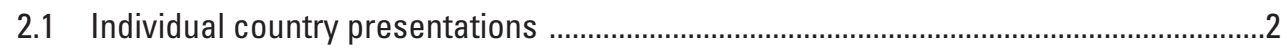

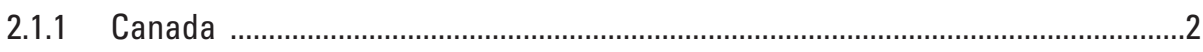

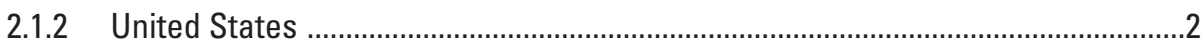

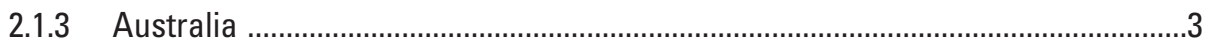

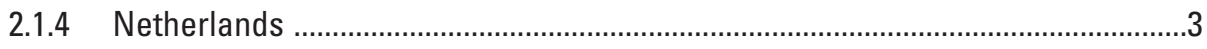

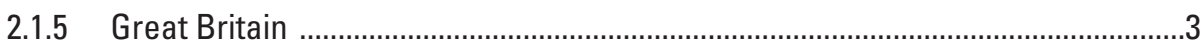

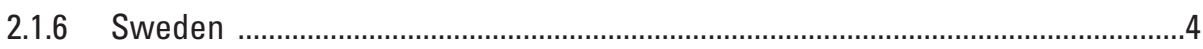

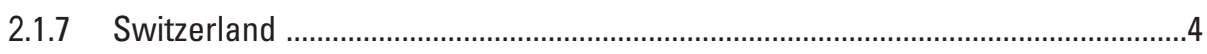

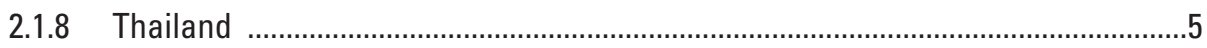

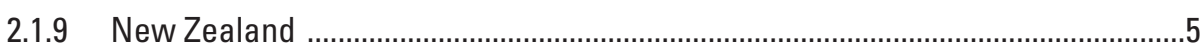

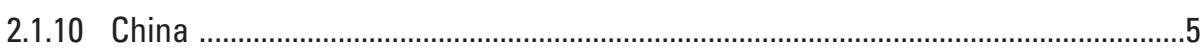

2.2 Goals, essential functions, successes, challenges, and needs ....................................

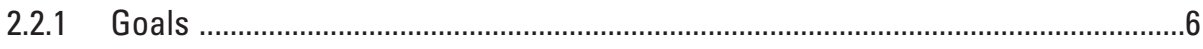

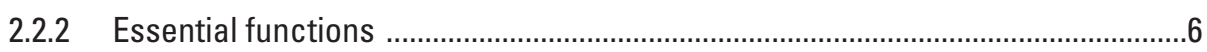

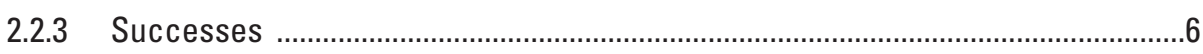

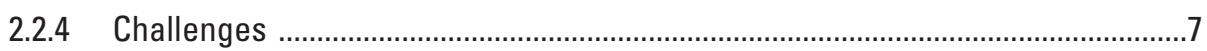

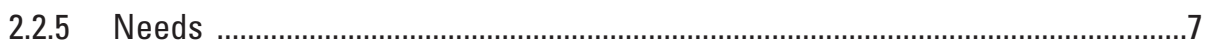

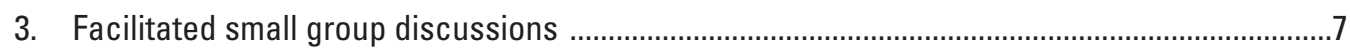

3.1 Essential goals and functions of a national wildlife health program ..............................7

3.2 Necessary capabilities and competencies of a national wild life health program ..........9

3.2.1 Capabilities that distinguish a national wildlife health program .........................

3.2.2 Management of a national wildlife health program .........................................10

3.2.3 Needs to meet this vision of a national wildlife health program ........................10

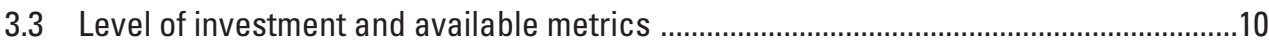

4. Plenary session on additional need for a national wildlife health program .............................12

5. Plenary session on key features of a national wildlife health program ...................................12

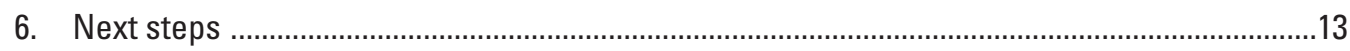

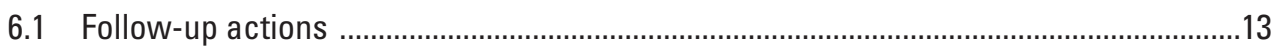

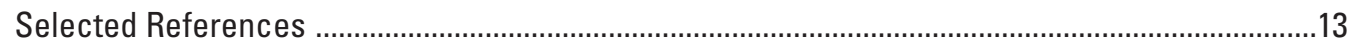

Appendixes

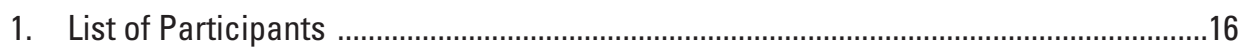

2. Workshop Program ...........................................................................................17

3. National Wildlife Health Programs Working Group Guidance ......................................18 


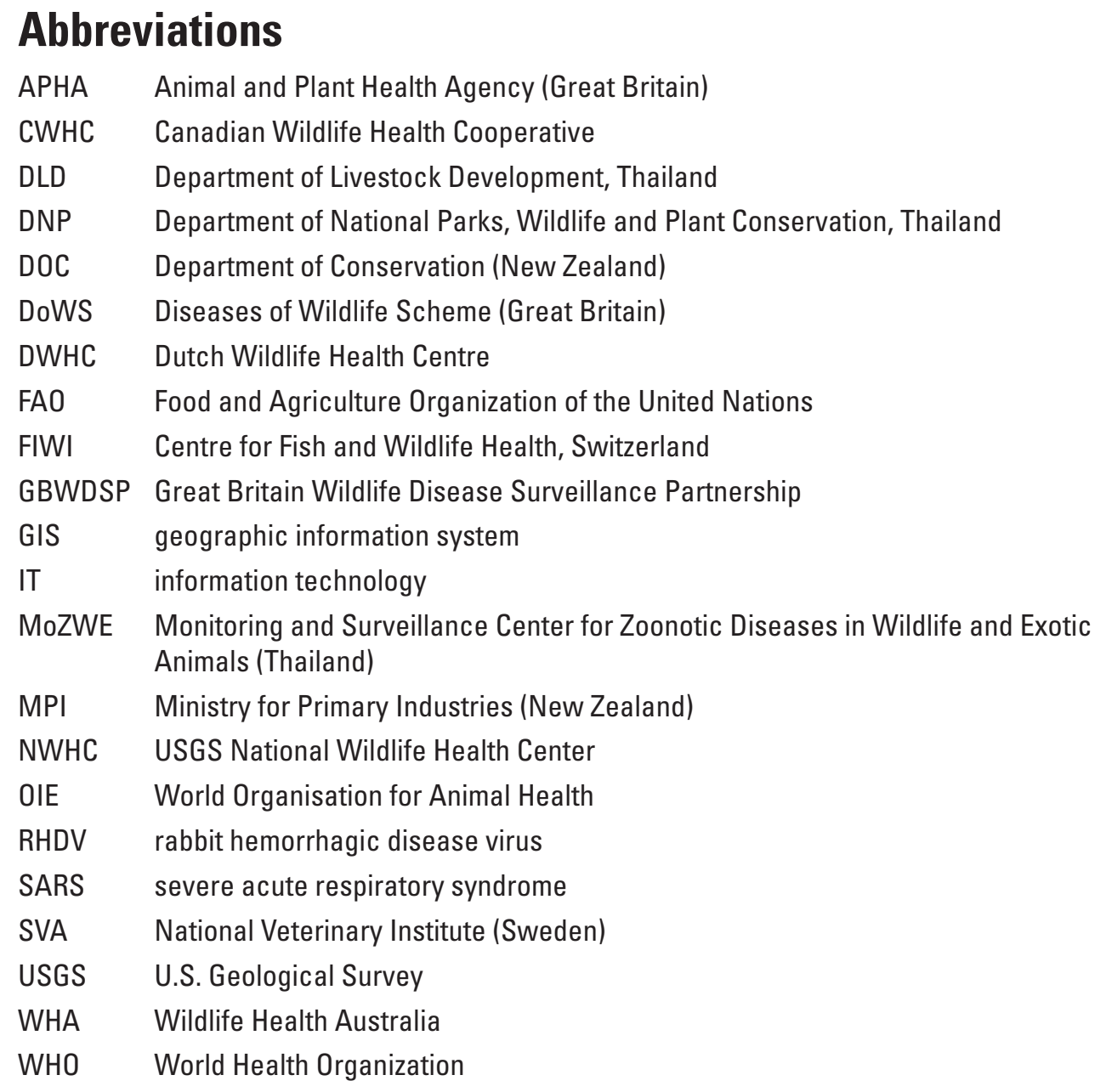




\title{
Report of the Workshop on Evidence-Based Design of National Wildlife Health Programs
}

\author{
By J. Paul Duff, Dolores Gavier-Widén, ${ }^{2}$ Tiggy Grillo, ${ }^{3}$ Hongxuan He, ${ }^{4}$ Hang Lee, ${ }^{5}$ Natalie T. Nguyen, ${ }^{6}$ \\ Parntep Ratanakorn, ${ }^{7}$ Jolianne M. Rijks, ${ }^{8}$ Marie-Pierre Ryser-Degiorgis, ${ }^{9}$ Jonathan M. Sleeman, ${ }^{6}$ \\ Craig Stephen, ${ }^{10}$ Toni Tana, $^{11}$ Marcela Uhart, ${ }^{12}$ and Patrick Zimmer ${ }^{10}$
}

\section{Introduction}

The workshop on evidence-based design of national wildlife health programs was organized and managed by Dr. Craig Stephen from the Canadian Wildlife Health Cooperative and Dr. Jonathan Sleeman from the U.S. Geological Survey, National Wildlife Health Center.

\subsection{Workshop objectives}

The overall objective was to use available evidence and selected subject matter expertise to define the essential functions of a National Wildlife Health Program and the resources needed to deliver a robust and reliable program, including the basic infrastructure, workforce, data and information systems, governance, organizational capacity, and essential features, such as wildlife disease surveillance, diagnostic services, and epidemiological investigation. We defined national wildlife health programs widely to include programs that address wildlife-associated infectious and non-infectious diseases that impact public health, livestock, wildlife health, and the environment. This workshop also provided the means to begin the process of defining the

\footnotetext{
${ }^{1}$ Animal and Plant Health Agency, Great Britain.

${ }^{2}$ National Veterinary Institute, Sweden.

${ }^{3}$ Wildlife Health Australia, Sydney, Australia.

${ }^{4}$ National Research Center for Wildlife Borne Diseases, Institute of Zoology, Chinese Academy of Sciences, China.

${ }^{5}$ Seoul National University College of Veterinary Medicine, Republic of

${ }^{6}$ USGS National Wildlife Health Center, USA.

${ }^{7}$ Mahidol University, Faculty of Veterinary Science, Thailand.

${ }^{8}$ Dutch Wildlife Health Centre, Netherlands.

${ }^{9}$ Centre for Fish and Wildlife Health, University of Bern, Switzerland.

${ }^{10}$ Canadian Wildlife Health Cooperative, Canada.

${ }^{11}$ Ministry for Primary Industries, New Zealand.

${ }^{12}$ University of California, Davis, USA.
} Korea. essential attributes of a national wildlife health program that could be scalable and adaptable to each nation's needs.

\subsection{Expected outcomes}

The expected outcomes of this workshop were to (1) describe a shared vision of a program's essential attributes; (2) provide justifications for the establishment and (or) ongoing operations of national wildlife health programs; and (3) develop a forum for a network of national wildlife health program managers. The desired output of this workshop was to produce a report that summarizes the expert opinions of national wildlife health program leaders and (or) managers that can assist countries or organizations working to justify, design, or explain critical capacities and resources needed for effective national wildlife health programs.

\subsection{Methods and target participants}

This working group meeting used a subject matter expert elicitation process to improve the understanding of the essential functions of a national wildlife health program and identify the knowledge gaps. A purposively selected small group was invited to facilitate the discussion and ensure international representation of individuals responsible for delivering national wildlife health programs. There are many individuals with expertise germane to this topic; however, the group of invited participants was deliberately kept small to encourage dialogue and ensure the meeting was of a manageable size and productive. The invited group included 14 participants consisting of wildlife managers, researchers, and veterinarians from 11 countries (Australia, Canada, China, the Netherlands, New Zealand, Republic of Korea, Sweden, Switzerland, Thailand, Great Britain, and the United States). Attendees also included some wildlife focal points of the World Organisation for Animal Health (OIE), an international organization; representatives from the Wildlife Disease Association, a non-profit organization; and one person with specialist knowledge of wildlife health programs in Latin America. Prior to the workshop, participants were provided 
information on the purpose of the workshop, the workshop agenda, and background materials on current national wildlife health programs. Minutes and group discussions were recorded by note takers.

The list of participants is provided as appendix 1 .

\subsection{Workshop program}

The workshop consisted of introductory presentations on the workshop purpose and anticipated outcomes as well as brief presentations on existing programs in Canada, the United States, Australia, the Netherlands, Great Britain, Sweden, Switzerland, Thailand, New Zealand, and China. The remainder of the workshop consisted of small group, facilitated discussions on the following topics: What are the essential functions, goals and objectives, and targeted knowledge users of a national wildlife health program? What are the necessary capabilities and competencies of a national wildlife health program? What is the necessary level of effort and investment to meet the goals and objectives? What metrics are available to justify ongoing and consistent investment? The small group discussions were followed by plenary sessions on the additional needs and key features of a national wildlife health program, concluded by a discussion of next steps and action items.

The program of the workshop is provided as appendix 2 .

\section{Introductory presentations}

\subsection{Individual country presentations}

Presentations were given regarding existing national wildlife health programs in different countries. The focus of each presentation included the goals, functions, successes, and programmatic challenges. The presenters in this session represented Canada, the United States, Australia, the Netherlands, Great Britain, Sweden, Switzerland, Thailand, New Zealand, and China. A brief summary of each presentation is provided below.

\subsubsection{Canada}

The Canadian Wildlife Health Cooperative (CWHC; $h t t p: / / w w w . c w h c-r c s f . c a /)$ has a vision of a world that is safe and sustainable for wildlife and society. Its mission is to promote and protect the health of wildlife and Canadians through leadership, partnership, investigation, and action. The CWHC is organized as a community of practice with a national office supporting and coordinating six regional centers in each of Canada's veterinary colleges and one provincial animal health laboratory. Fifteen full-time staff, 11 part-time staff, and up to 50 students deliver CWHC services using a budget of approximately $\$ 3.1$ million
Canadian dollars derived from more than 36 different funders. Half of the budget supports monitoring and surveillance activities, 19 percent supports knowledge mobilization, 17 percent supports program management, and 12 percent supports assessment activities.

The CWHC is a successful model of the delivery of a national wildlife health program based on four criteria: (1) it has been sustainable for 25 years; (2) it is a trusted voice for independent science advice across sectors; (3) it has advanced harmonization of approaches to wildlife health across Canada; and (4) it has provided significant contributions to wildlife health capacity through training, research, and development. Significant challenges complicate further advancement, namely the challenge of inspiring ongoing or increased funding using a program based largely on finding problems rather than generating solutions, balancing the multiple needs and priorities of many funders, and promoting and sustaining a national approach in the absence of a national strategy.

\subsubsection{United States}

The U.S. Geological Survey (USGS) National Wildlife Health Center's (NWHC; http://www.nwhc.usgs.gov/) mission is to safeguard wildlife and ecosystem health through dynamic partnerships and exceptional science. It is a U.S. Federal Government research center with approximately 120 staff, students, and volunteers. It operates nationally and has an increasingly international outreach as a consequence of a number of requests to assist other nations create their own national wildlife health centers or programs. The primary facility which includes Biosafety Level-3+ diagnostic and research laboratories is based in Madison, Wisconsin, and a field station in Honolulu, Hawaii, covers the Hawaiian Islands and the Pacific region.

The NWHC achieves its mission by conducting in-depth epidemiological investigations of wildlife disease outbreaks, responding to animal health emergencies, leading and participating in national-level targeted surveillance for high-consequence wildlife-associated pathogens, performing diagnostic evaluations including pathogen discovery and characterization, and conducting applied research including disease ecology and modeling, infectious disease research, and development of management tools, especially orally delivered vaccines. The NWHC has successfully delivered these services for more than 40 years and has generated a considerable dataset on wildlife mortality events, contributed to the discovery of pathogens such as West Nile virus and Pseudogymnoascus destructans, the causative agent of whitenose syndrome in bats, and has an active, diverse research program.

Current challenges for the NWHC include increasing requests for services and the need to maintain and modernize the current facilities. Additional challenges for a national wildlife health program in the United States include fragmentation of the wildlife health community with responsibilities divided among State agencies, regional 
entities, and various Federal agencies, and inadequate coordination among all parties.

\subsubsection{Australia}

Wildlife Health Australia (WHA; https://www. wildlifehealthaustralia.com.au/) activities focus on the increasing risk of emerging and emergency diseases that can affect wild animals and impact Australia's biodiversity, human health, trade, and tourism. WHA provides the framework for Australia's general wildlife health surveillance system to assist Australia in better identifying, assessing, articulating, and managing these risks. Key functions include maintenance of the national wildlife health information and database; coordination of the national wildlife health network, support for prevention, preparedness, and response to wildlife diseases; and intelligence gathering and dissemination to inform decision making and capacity building through communication, education, and training.

WHA is a not-for-profit association initiated by the Australian Government. Core funding is from the Australian Department of Agriculture and Water Resources and is matched by significant in-kind contributions from the States and Territories, private veterinarians, zoo-based veterinarians, universities, and others, leveraging the cash contribution of the Australian Government approximately five-fold. Five fulltime and two part-time staff administer and coordinate WHA's projects and programs in collaboration with more than 38 core partner agencies and organizations and more than 600 WHA members across biosecurity, human health, and environmental sectors.

Success includes integration of WHA activities into Australia's biosecurity arrangements for more than 12 years, maintaining the trust and confidence of governmental and nongovernmental stakeholders, facilitating effective, crossdisciplinary multi-agency networks to generate norms and standards for wildlife health and disease monitoring, and maintaining a complete picture of wildlife health information for Australia.

Challenges include communicating the value of wildlife health to all levels of government and funding bodies, quantifying the value of maintaining an engaged network of wildlife health expertise, adapting and growing surveillance networks, and sustaining core funding.

\subsubsection{Netherlands}

The purpose of the Dutch Wildlife Health Centre (DWHC; https://www.dwhc.nl/) is to enhance knowledge of and expertise in wildlife health in the Netherlands, in view of providing scientifically based information for political and practical decisions concerning public health, wild and domestic animal health, and nature conservation issues. The DWHC performs wildlife disease surveillance and diagnostic activities including investigation of approximately
500 unusual wildlife mortality incidents per year with associated data- and tissue-banking, early warning activities, and research. The DWHC also provides expert advice and information on wildlife diseases in the Netherlands.

The DWHC has been embedded in the Pathology Department of the Faculty of Veterinary Medicine, Utrecht University, since 2008. It receives baseline financial support from the Dutch Ministry of Economic Affairs, the Dutch Ministry of Public Health, Welfare and Sports, and Utrecht University. Baseline funding covers personnel (4.5 full-time employees) and material costs in a ratio of 70:30. Funding for research must be acquired. The DWHC operates in close collaboration with a large network of field and diagnostic organizations, which is crucial for its success.

Among the achievements of the Center and its network is the detection of zoonotic and wildlife diseases previously considered absent in the Netherlands, such as ranavirus infections, tularemia, and tick-borne encephalitis, as well as public access to comprehensive up-to-date information on wildlife health issues relevant to the Netherlands.

Internal organizational challenges are capacity for pathogen detection and identification, information management, and timely analysis and communication. External organizational challenges are assurance of continuity, visibility versus capacity, and sector differences in view of importance and management of wildlife diseases.

\subsubsection{Great Britain}

Since 1998 the Diseases of Wildlife Scheme (DoWS), centered around the network of government veterinary laboratories, has delivered surveillance for wildlife diseases in England and Wales to the British government. In 2009, the England Wildlife Health Strategy (Department for Environment, Food and Rural Affairs, 2009) was produced to guide the government in their approaches to wildlife disease, and one of the Strategy's recommendations was that the Veterinary Laboratory Agency, now a part of the Animal and Plant Health Agency (APHA), would chair a partnership of seven governmental and non-governmental organizations to provide wildlife disease surveillance for Great Britain (England, Scotland, and Wales). This is now known as the Great Britain Wildlife Disease Surveillance Partnership (GBWDSP) and it comprises the APHA DoWS (as Chair), Scottish Agricultural Colleges Consulting, the Centre for Environment, Fisheries and Aquaculture Science, the Forestry Commission England, the Institute of Zoology, the Wildfowl and Wetlands Trust, and Natural England.

The functions of the DoWS are to detect and characterize wildlife disease threats and report these directly to government. Specifically this includes wildlife diseases that are new and re-emerging threats to human health, threats to livestock health, incursions of exotic disease, indicators of environmental change and pollution, and threats to biodiversity. 
The APHA currently has eight veterinary laboratories including a central research facility (Weybridge) in Great Britain. These laboratories have cutting-edge diagnostic capabilities in infectious pathogens, non-infectious agents, and toxins, and the APHA itself employs wildlife ecologists, epidemiologists, and disease modelers. Identified disease threats are disseminated and escalated through well-defined pathways to the government.

Recent collaborations have increased successes of the DoWS. The DoWS has produced the first reports and assessments of new diseases such as bovine tuberculosis in wild boar and common seals; highly pathogenic avian influenza in wild waterbirds; white-nose syndrome in an English bat; Echinococcus multilocularis; and rabbit hemorrhagic disease virus (RHDV), RHDV2, and European brown hare syndrome viruses in wild leporids. Current challenges include maintaining funding streams, filling gaps in surveillance networks, maintaining expertise, and collecting carcasses from certain localities.

\subsubsection{Sweden}

The Swedish National Wildlife Disease Surveillance Programme aims at obtaining information about the disease situation in wild animals in Sweden through comprehensive and structured monitoring, analysis, and reporting. The resulting knowledge can be used for intervention and management of wild animal populations, game animals, and environmental monitoring.

The Programme is conducted by the Division of Wildlife, Department of Pathology and Wildlife Diseases, at the National Veterinary Institute (SVA), Uppsala, Sweden. The SVA has approximately 350 employees. The infrastructure consists of three Biosafety Level-3 microbiology laboratories; a Biosafety Level-2 and Biosafety Level-3 post mortem room; pathology, bacteriology, virology, parasitology, molecular diagnostics, and chemistry diagnostic laboratories; an epidemiology department; and a communications department. The staff at the Division of Wildlife consists of six full-time pathologists, one full-time biologist, technicians, laboratory assistants, and secretaries. The collection of reports from the field is done through the website, by email, by telephone, and through an app for smartphones, which is under development. A network of sample providers, organized in conjunction with the Swedish Hunting Association, contributes to the collection of samples, as well as the general public, researchers, and others who also submit carcasses and samples for examination.

Successes include the identification of new diseases, contributions to risk assessments for public health and wildlife conservation, and the development and maintenance of a frozen tissues bank with more than 50,000 samples associated with pathology and animal data that have been collected since the early 1980s. A report of the Wildlife Disease Surveillance conducted in Sweden in 2015 can be found at http://www.sva. se/globalassets/redesign2011/pdf/om_sva/publikationer/vildadjur/Wildlife-disease-monitor-2015.pdf.

Some of the main programmatic challenges include the use of a large part of the resources by the targeted programs, scarcity of trained pathologists, difficulties in obtaining and transporting carcasses of wild animals from remote areas, and limited field work.

\subsubsection{Switzerland}

The Centre for Fish and Wildlife Health (FIWI; http:// www.fiwi.vetsuisse.unibe.ch) acts as the national competence center for wildlife diseases on the mandate of the Swiss Federal authorities. It has developed from a small diagnostic unit to a more comprehensive national program over the past 60 years, moving from pathological studies to disease ecology. The mandate includes four main activities: disease diagnostics (general health surveillance), research (mainly on conservation issues or challenges at the wildlife/livestock interface), consulting, and teaching (including training of field partners).

The mandate of the Federal Office of Environment (the Swiss environmental agency) is based on article 11.2 of the Federal ordinance regarding hunting and protection of freeranging mammals and birds, and stipulates that the Federal Office of Environment has to support (within the available budget) applied research in wildlife biology and ornithology; in particular, investigations into species conservation, deterioration of habitats, damage due to wildlife, and diseases of wildlife. The FIWI is a unit of the Vetsuisse Faculty of the University of Bern. The FIWI Wildlife Group consists of 4-5 part-time employees (veterinarians and a biologist), 2-3 parttime technicians, and 3-4 postgraduate students. Infrastructure (including offices, necropsy room, histology laboratory, other laboratories, storage rooms and freezers, general secretariat, IT and other technical support, and vehicles) is provided by the University. Most of the funding for salaries and consumables is provided by the Federal government, via a contract renewable every 4 years, and additional contracts for specific time-limited services or research projects.

Successes include the increasing geographical coverage and expanding partner network, the detection of numerous diseases and pathogens not previously known to occur in Switzerland or elsewhere, increasing governmental recognition of the importance of the national program, involvement in the Federal early warning strategy, repeated requests for wildlife pathogen data to develop livestock disease control programs, a steadily growing biobank, and increasing requests for training by various stakeholders. Main challenges are personnel turnover; absence of a long-term strategy due to the lack of a long-term contract and budget; insufficient work facilities and staff; and lack of recognition of the importance of a group dedicated to wildlife health by the University faculty. 


\subsubsection{Thailand}

The Monitoring and Surveillance Center for Zoonotic Diseases in Wildlife and Exotic Animals (MoZWE), Faculty of Veterinary Science, Mahidol University, Thailand, was established in 2004 with approval by the Thailand cabinet for the monitoring and warning of outbreaks of avian influenza and other diseases in wildlife. The center collaborates with the Department of National Parks, Wildlife and Plant Conservation (DNP), Department of Livestock Development (DLD), and Department of Disease Control to perform disease surveillance in wildlife, captive birds in zoos, native or migratory species of free-ranging birds in Thailand, and other species of animals. Various samples of wildlife, collected by MoZWE, DNP, law enforcement agencies, zoos, universityaffiliated scientists, and others, are sent to the MoZWE laboratory for diagnosis of wildlife diseases. Through the end of December 2015, about 50,000 samples from various species of animals including wildlife, zoo animals, and domestic animals were investigated for pathogens by MoZWE's laboratory. The data from disease surveillance and laboratory testing were collected and used in the disease control program to immediately notify the government organization that has competent authority to implement appropriate responses for disease control. MoZWE is dedicated to assessing the impact of zoonotic diseases and diseases of wildlife, and identifying the role of various pathogens in contributing to wildlife losses. In addition, MoZWE is a designated Food and Agriculture Organization (FAO) of the United Nations Reference Centre for Zoonotic and Wildlife Diseases and collaborates with FAO and other international partners to provide assistance and expertise in preventing and detecting transboundary animal diseases, including zoonoses, and improving risk and disease management.

As a department of the faculty of veterinary science at Mahidol University, MoZWE is composed of four units, which are wildlife epidemiology, laboratory diagnosis with an Animal Biosafety Level-3 facility, quality control, and an administrative unit. For these reasons, the Thai cabinet agreed to establish the National Wildlife Health Center at the Faculty of Veterinary Science, Mahidol University. The responsibilities of the center are to monitor, survey, and warn of the threats against wildlife and environmental health and (or) diseases originating from wild animals and emerging diseases in order to ultimately strengthen the community and ecological well-being.

\subsubsection{New Zealand}

The purpose of the Ministry for Primary Industries (MPI) wildlife surveillance program in New Zealand is to facilitate early detection of exotic and emerging diseases, support New Zealand's statements of freedom from specific pests and diseases, provide baseline information on endemic disease occurrence in New Zealand's wildlife, and support New Zealand's international reporting obligations.

The program is integrated into the national animal surveillance system, incorporating nationwide general passive surveillance as well as complementary targeted surveillance for specified diseases in wildlife. A national exotic pest and disease notification system provides centralized 24/7 reporting and investigation of unusual disease events in all animals in New Zealand, including wildlife (Tana, 2014). Investigations are managed by trained disease investigators and are supplemented by a national network of veterinary investigators, which enables appropriate investigations of disease events in wildlife that are identified by any organization or individuals in New Zealand. Diagnostic capability is provided by the National Animal Health Laboratory.

The program also collates wildlife occurrence data created by the MPI and other individuals and organizations undertaking surveillance or working with wildlife, particularly the New Zealand Department of Conservation (DOC).

Successes include the consolidation of wildlife disease surveillance within an existing system, which provided efficiency gains, prevented duplication of resources, and provided consistency with the One Health approach; an increase in the profile of wildlife surveillance; improved quality of data collection; and collaboration with other government agencies undertaking wildlife disease surveillance.

Key challenges include relationship management and communication, privacy and intellectual property protection for contributors of information, and a lack of standardized physiological reference lists and validated diagnostic tests for many species.

\subsubsection{China}

In China, a surveillance system of wildlife diseases has been established since 2005 in response to the 2003 severe acute respiratory syndrome (SARS) outbreak. Currently, it has 350 national monitoring stations, 768 provincial monitoring stations, and more than 2,000 monitoring stations at the county level. The National Research Center for Wildlife Borne Diseases is the main Technical Support Unit under the system.

The surveillance system has been successful. Measures have been widely used for wildlife disease surveillance and management, including a remote sensing monitoring system, routine reporting (including negative findings), data submission, and information systems. Moreover, a platform for wildlife-borne diseases, including models for pathogen isolation, molecular analysis, rapid detection, and genomic research, has been established in order to better respond to wildlife-related events and outbreaks. 
Many wildlife disease events are dealt with under collaborations with other departments, institutes, and universities. International collaborations have been developed as well. The Asia-Pacific Wildlife Diseases Network and annual workshops on Regional Surveillance and Research for Wildlife-Borne Diseases were initiated by the Chinese Academy of Sciences and the U.S. Department of Agriculture in 2010. The members of the network are from more than 10 countries in the Asia-Pacific region and several international organizations.

The surveillance system also faces some challenges. Factors regulating disease emergence and spread are multifaceted, and disease emergence is global and complex. While specific factors that give rise to infectious disease may differ, diseases in all sectors arise from the interactions of people, domestic animals, and wildlife with the environment, so collaboration is needed among different countries and organizations.

\subsection{Goals, essential functions, successes, challenges, and needs}

The goals, essential functions, successes, challenges, and needs identified in this section were gathered from the national wildlife health program presentations and follow-up discussions from the whole group. All national wildlife health programs included in the presentations were in different stages of development and maturity and operated on different geographical scales and organizational structures. Organizational structures presented varied and included; dedicated centers and (or) partnership arrangements that involved non-government and (or) governmental organizations, and national programs delivered by government systems. Therefore, it is understood that the following ideas discussed may not be unconditionally generalizable.

\subsubsection{Goals}

The goals of these national wildlife health programs that participants commonly identified included:

- Protecting wildlife and ecosystem health from the negative impacts of wildlife diseases and emerging infectious diseases;

- Early warning of emerging and re-emerging wildlife diseases of zoonotic and (or) agricultural concern;

- Conducting wildlife disease monitoring and surveillance on a national level;

- Obtaining, maintaining, and disseminating scientifically based information on wildlife health, diseases, and conservation to governmental and nongovernmental partners, policy makers, and the general public; and
- Providing and enhancing knowledge and expertise in wildlife health and diseases on a national level.

\subsubsection{Essential functions}

The functions of these national wildlife health programs that participants commonly identified included:

- National wildlife disease monitoring and surveillance;

- Data management, including the collection, analysis, and dissemination of information on wildlife diseases;

- Pathogen detection, discovery, and characterization;

- Determination of freedom from wildlife diseases of regulatory concern;

- Expert advice for wildlife management and decision making;

- Networking, coordination, and harmonization of standards and efforts on national and international levels;

- Early warning of and response to wildlife diseases, emerging and re-emerging infectious diseases, zoonotic diseases, agricultural diseases, and diseases of wildlife conservation concern;

- Fill knowledge gaps through targeted surveillance and applied research;

- Risk assessments for wildlife diseases;

- Provision of information to stakeholders for the purpose of a One Health approach to disease prevention and control;

- Communication and outreach, including risk communication, dissemination of information, and raising awareness of wildlife diseases, on local and national levels; and

- Education and training for wildlife researchers and managers.

\subsubsection{Successes}

The successes of these national wildlife health programs that participants commonly identified included:

- Growth and maintenance of programs and capacities on a national level;

- Development of trust and increased recognition of the importance of wildlife health and surveillance systems from governmental and nongovernmental partners and stakeholders; 
- Discovery and early detection of emerging and re-emerging wildlife diseases;

- Development and improvement of diagnostic tools, technology, and skill sets specific to wildlife disease detection;

- Development of national disease prevention, management, and response plans through multi-agency frameworks;

- Development of data management tools and improvement of data quality;

- Contributions to risk assessments for public health, agriculture, and wildlife conservation;

- Establishment of sample repositories or biobanks; and

- One Health approaches for the protection of human, animal, and environmental health.

\subsubsection{Challenges}

The challenges of these national wildlife health programs that participants commonly identified included:

- Concerns about the sustainability of the programs due to uncertain funding and inadequate facilities despite growing needs and the increasing number of projects;

- The high level of investment needed for quality passive and active surveillance;

- Personnel turnover and loss of expertise;

- Competition for limited funding and (or) the need to find multiple funding sources that may have differing values or priorities compared to those of the national wildlife health program;

- Lack of awareness and (or) understanding by stakeholders and decision makers of the goals and objectives of a national wildlife health program;

- Fragmentation and lack of coordination on the delivery of services due to an absence of legislative mandate for most countries and the division of responsibilities, or governance structures;

- Lack of standardized case definitions specific to wildlife health and disease;

- Lack of validated diagnostic techniques specific to wildlife health and disease;

- Legislative complexities regarding data privacy, intellectual property, and the release of information; and
- Maintenance of a visible program and network where samples are received/submitted for wildlife disease surveillance and investigation.

\subsubsection{Needs}

The needs of these national wildlife health programs that participants commonly identified included:

- Skilled leadership and well-trained personnel through continuing education and training;

- Improved networking, communication, and coordination of activities; and

- Enhanced general (passive) and targeted (active) surveillance capacities, diagnostic capabilities, data management tools, biobanking, and epidemiology and pathology expertise.

\section{Facilitated small group discussions}

The attendees of the meeting divided into three groups of four to five participants to answer a set of questions. Each small group spent about 1 hour discussing the questions and then returned to the whole group to discuss the answers. The following summaries are taken from the small group discussions along with any additional remarks made by the larger group.

\subsection{Essential goals and functions of a national wildlife health program}

\section{Dr. Jonathan Sleeman (Facilitator), Dr. Natalie Nguyen (Note taker)}

The first set of questions discussed was "What are the essential functions of a national wildlife health program? For those functions, what are the associated goals and objectives? Who are targeted knowledge users?"

The participants identified the need for an overarching mission statement that would encompass the wide range of existing and developing national wildlife health programs, independent of a program's capacities and resources. An example of this overarching mission statement that was developed was "To protect the health of humans, livestock, ecosystems, and wildlife from wildlife-associated diseases." Because different countries have varying governance structures, needs, and capacities, the group noted that it is important to simultaneously consider the scope of practice of the national wildlife health program and the beneficiaries when applying this mission statement. The participants 
identified 11 core goals and functions of a national wildlife health program, which could be achieved through a variety of organizational structures. There is no particular order to the goals and functions listed because the level of importance of each will depend on the national program's overarching goals and objectives. For each core goal and function, the targeted knowledge users were identified. The 11 core goals and functions identified were as follows.

\section{Goal 1: Perform wildlife disease surveillance.}

Functions: Threat and hazard identification, early warning and detection of emerging and reemerging diseases, assurance of freedom from diseases of regulatory concern, identification of presence and absence of diseases, and assessment of the successes of management programs.

Capabilities: Diagnostic capabilities, diagnostic test development, epidemiological monitoring and assessment of wildlife diseases, and biostatistics.

Targeted knowledge users: Public health authorities, agriculture ministries, wildlife environment/conservation agencies, and other wildlife stakeholders (industry, agriculture, researchers, hunters, wildlife rehabilitators, and the general public).

Goal 2: Determine cause of wildlife morbidity and mortality.

Functions: Diagnostic and epidemiological investigations.

Capabilities: Network of field-based personnel, best available diagnostic techniques, genetic sequencing, and validated tests, as well as surge capacity in the event of a large-scale outbreak.

Targeted knowledge users: Wildlife environment/ conservation agencies, public health authorities, agriculture ministries, and the research community.

Goal 3: Prevent the introduction and spread of wildlife diseases.

Functions: Disease control/management; development of emergency response plans; human, domestic, and wild animal health protection from wildlifeassociated diseases; control of disease vectors and reservoirs; eradication of wildlife diseases; and dissemination of information and advice to regional, national, and international organizations and agencies for the prevention of transboundary movement of disease.

Targeted knowledge users: Government public health authorities, agriculture ministries, wildlife environment/conservation agencies, and non-profit organizations.
Goal 4: Provide expert consultation to the government and other stakeholders on wildlife disease issues.

Functions: Provision of wildlife disease expertise and advice from wildlife health experts. This was considered a key function of a national wildlife health program.

Capabilities: Including but not limited to pathologists, epidemiologists, microbiologists, virologists, parasitologists, veterinarians, researchers, and other biologists.

Targeted knowledge users: Governmental and nongovernmental agencies, policy makers, wildlife environment/conservation agencies, and the general public.

Goal 5: Obtain wildlife health data from sources within the country to support evidence-based action and support the information provided to government agencies, policy makers, and other members of the wildlife community.

Functions: Development and maintenance of databases, data sharing agreements, and data management, which allow information to be stored, retrieved, analyzed, and disseminated, and used for epidemiological analyses. These national databases are critical for reporting on wildlife health status, a critical expectation of the OIE.

Capabilities: Health information management, including data storage, analysis, and communication.

Targeted knowledge users: Governmental agencies, researchers, and the general public.

Goal 6: Provide specialist knowledge of hazard identification, characterization, and potential consequence to stakeholders and policy makers to make informed decisions.

Functions: Risk assessment and risk communication, which includes assisting in the management of specific diseases.

Capabilities: Risk analysis encompassing risk assessment, management, and communication.

Targeted knowledge users: Policy makers, the general public, and specialized groups in agriculture and other sectors.

Goal 7: Harmonize and standardize efforts and activities, and maximize coverage of a nation.

Functions: National, international, and One Health coordination of activities.

Capabilities: Team management and leadership skills, facilitation and communications skills, and epidemiological capacity. 
Targeted knowledge users: Policy makers, the general public, and governmental and nongovernmental agencies, including State and Federal public health, conservation, and agricultural officials and international organizations such as the OIE, World Health Organization (WHO), and FAO.

Goal 8: Provide retrospective analyses of pathogens, traceability of pathogens, data on the presence and absence of pathogens, and reference collection of materials, including seed stocks and cell cultures.

Functions: Provide historical records and materials for research and to support claims of disease freedom or analysis of spread.

Capabilities: Biobanking and microbiological research capacity.

Targeted knowledge users: Scientific and research communities and agriculture ministries.

Goal 9: Share knowledge, develop trust, and maintain a network among stakeholders, in addition to informing and advising the public of wildlife diseases.

Functions: Communication and outreach of wildlife diseases to stakeholders through websites, social media, reports, and media releases.

Capabilities: Team management, leadership, and communications skills.

Targeted knowledge users: Wildlife environment/ conservation agencies and the general public.

Goal 10: Plan for succession, long-term strategic planning, increase awareness of wildlife disease prevention and management, and maintain and develop wildlife health expertise.

Functions: Human resource planning and provision of education and training opportunities to maintain a skilled workforce.

Capabilities: Strategic planning, administration and education expertise.

Targeted knowledge users: Recipients of this education.

Goal 11: Understand the epidemiology and ecology of wildlife diseases and develop disease management tools.

Functions: Applied wildlife research focused on longitudinal and prospective studies, in addition to basic research. This was not a unanimously identified function.
Capabilities: Wildlife epidemiology and ecology, infectious disease research, genetics, immunology, and vaccinology.

Targeted knowledge users: General public, specialized groups in agriculture and hunting, the scientific research community, and governmental agencies.

With all these goals and functions, the participants of the workshop unanimously agreed that it is important to define and keep in mind the mission of each individual program as these goals are pursued. The group agreed that it is important to improve and create awareness of wildlife diseases and existing national wildlife health programs, play an advocacy role, protect, conserve, and promote animal, environmental, and human health, and always continue to be strategic, forward-thinking, and continuously improve and develop these national wildlife health programs.

\subsection{Necessary capabilities and competencies of a national wildlife health program}

\section{Dr. Craig Stephen (Facilitator), Dr. Marie-Pierre Ryser- Degiorgis (Note taker)}

The question discussed by this group was "What are the necessary capabilities and competencies of a national wildlife health program?" The answer to this question was broken down into three sections: What are the capabilities that distinguish a national wildlife health program? What should proper organizational management include? What is needed to meet this vision of a national wildlife health program? In this group's discussion, there was some overlap between the identified capabilities and the essential goals and functions of a national wildlife health program that are stated above.

\subsubsection{Capabilities that distinguish a national wildlife health program}

Several factors were identified that were believed to distinguish a national wildlife program. First, it was discussed that one of the unique capabilities of a national wildlife program was to deliver an integrated program on all aspects of the surveillance cycle, including case detection, field collection of samples, diagnostics, analysis and assessment, development of surveillance plans and reports, and communication of the importance of wildlife health to reflect multiple geographic scales, communities, and priorities in a country. Second, it was discussed that national programs may have the unique ability of hazard detection of pathogens and pollutants, which requires specialized wildlife disease pathologists and biologists, diagnostic laboratory capabilities specific to wildlife with proper quality assurance, case flow and sample collection, access to specimens by the program and their partners, and specialized wildlife disease research to develop credibility, a good reputation, and trust among partners. It was identified that a national wildlife 
health program provides tools, techniques, and networks of expertise specific to wildlife that may not be developed by other sectors. Additionally, it was identified that the ability to provide threat/risk assessments and support response on a national and international scale is also unique to a national program. Threats and risk assessments require knowledge of current animal populations, current and emerging wildlife disease concerns, knowledge of the governmental policies and priorities, and the ability to perform and mobilize field and diagnostic capacity. It was also discussed that national programs provide the ability for two-way communication between local and regional authorities and national authorities and serve as a central source of information that can help identify national priorities and translate information into policy. The ability to document and communicate the relevance of wildlife diseases appropriately was identified to require an understanding of the sensitivities and perceptions of wildlife disease and populations by stakeholders and the general public, especially when detection of a disease has the potential to lead to an adverse perception of wildlife species. It was agreed that trust between stakeholders and the national program, as well as a good reputation, are important for building relationships with partners, which allows for better integrative ability and interpretation of data with consideration of a larger picture. Another proposed key feature that was identified for a national program to have is a certain degree of legislated or delegated authority on wildlife healthrelated issues that is appropriate for the scale of the program. This legislated or delegated authority may range from full legislative authority to make decisions and act, the clear mandate from governmental authorities to deliver legislative functions on their behalf, or at minimum, participation in the development and review of national wildlife health strategies and actions. Proper leadership and the institutional management necessary to allow the program to function effectively were identified to underlie the knowledge, ability, and expertise that distinguish a national wildlife program.

\subsubsection{Management of a national wildlife health program}

The participants discussed that proper management of a national wildlife program requires the appropriate interpersonal skills, networks, information management, information sources, and communications. The appropriate interpersonal skills include good leadership and the ability to facilitate, listen, form teams, and manage conflict.

Networking was identified to include the ability to maintain a visible program where samples are received and submitted, hear priorities from outside sources, obtain the knowledge of wild population and pathogen dynamics, and build trust among partners. Information management was identified to include the ability to perform integrated analyses, manage and integrate data, execute proper information technology (IT) capabilities, and communicate information tailored to stakeholders. Information sources were identified to include the ability to obtain information from wildlife disease cases, diagnostics, populations, and on-the-ground responses. Lastly, it was discussed that a national wildlife health program needs the right personnel with proper communication skills to implement the strategy, develop and maintain trust among stakeholders, and communicate relevance of wildlife diseases.

\subsubsection{Needs to meet this vision of a national wildlife health program}

In order to meet the above vision of a national wildlife health program, the participants identified several key aspects that would be needed, including the personnel, infrastructure, relationships, legislation or official support to exist, and the ability to integrate the knowledge obtained. Some of the personnel identified as part of a national wildlife health program included, but were not limited to, pathologists, epidemiologists, biologists, and ecologists specialized in wildlife diseases, as well as proper management, administrative, and IT staff, and staff with communication, coordination, and geographic information system (GIS) skills. Additionally, appropriate infrastructure that was identified as important included diagnostic facilities for all hazards, storage for information and samples, IT capabilities, and a strategy to receive cases and perform field work and response. Relationships that were identified as needed included formal partnerships and professional networks in addition to informal relationships with the general public and the media. Additionally, legislation or official support to exist as a national wildlife health program was identified as important in order to provide a mandate to conduct the work. Lastly, the ability to integrate information in a manner that allows all the knowledge obtained to form a complete picture was identified as necessary.

\subsection{Level of investment and available metrics}

\section{Dr. Dolores Gavier-Widén (Facilitator), Dr. Marcela Uhart (Note taker) \\ The third set of questions discussed by the group was} "What is the necessary level of effort and investment to meet the goals and objectives? What metrics are available to justify ongoing and consistent investment in the program?" The group identified several points to be considered when a national wildlife program decides what level of investment is needed. The group's discussion focused mainly on wildlife disease surveillance because this was identified as a principal role for a national wildlife health program. First, the participants discussed that the goals of the surveillance program should be defined as well as the scope, beneficiaries, and partnerships of the surveillance work. Next, the group agreed that the geographic and species distribution to be covered by the program, which may depend on risk assessments, the current infrastructure and systems already in place in the country, and 
the existing networks and co-investments, should be identified. The group came to the agreement that passive surveillance was the minimal amount of effort needed for basic monitoring. It was also agreed upon that each national program should consider the target number of samples to include in their active surveillance. The next steps of investment and effort identified included other stages of the disease investigation process including reporting from the field, examining cases and diagnostic evaluation, assessing the impact, providing expert advice and communication to the public, providing education and training, and coordinating with internal and external partners. It was pointed out that coordination with partners can often consume significant funds, and may also at times be neglected. Lastly, the group agreed that it is important to ensure that a national wildlife health program is comprehensive and meets the needs of multiple sectors of human, agriculture, and wildlife health.

The group discussed possible metrics of success of a national wildlife health program, which occasionally included more than simple quantifiable results. The simplest metrics to measure the scientific success of a program that were identified included:

- Number of samples received;

- Number of cases examined and (or) diagnosed;

- Number of species covered;

- Number of positive cases identified;

- Maps of epidemiological analyses;

- Geographic coverage of services;

- Number of requests for information;

- Number of research publications and other scientific productions; and

- New funding obtained.

Some metrics to measure a program's network that were identified included:

- Measurements of the size of the network;

- Number of partners involved with the program; and

- Length of partnership arrangements and level of partnership engagement.

Likewise the simplest metrics to analyze communication success that were identified included:

- Number of visits to the program's website;

- Number of mentions of the program in media and press releases;

- Number of communications given to the public; and

- Number of scientific products.
Additional metrics that may also be used included:

- Commodity trade value in the absence of disease;

- Program's contribution to the economic value of hunting and tourism;

- Cost of early versus late detection, cleanup, and restoration in disease or contaminant situations;

- Cost of an epidemic that may have been potentially prevented; and

- Financial savings of the presence of a national wildlife health program that uses rationalized and harmonized approaches to prevent outbreaks and the associated costs.

The limitation of these metrics that was discussed is that these metrics may not always indicate the actual capacities and capabilities of a national wildlife health program. For example, if focus is on the number of cases received, and from year to year this number decreases or varies, this may be misinterpreted as a negative indication of the success of the program.

Outputs and outcomes that were identified that may be more difficult to measure included:

- Impact of prevention of disease;

- Cost/benefit ratio;

- Improved food security;

- Number of human lives saved;

- Sentinels for human disease, such as yellow fever in howler monkeys and West Nile virus in corvids;

- Hunting and recreational and cultural quality of life preserved;

- Conservation impact at the population level;

- Ecosystem services;

- Preparedness for rapid response and disease containment;

- Expert advice and support for the government;

- Impact on public health;

- Assurance of the absence of disease; and

- Social return on investment.

To address these more difficult metrics, the group agreed that it may be beneficial to analyze the metrics used by the agriculture and public health sectors as a parallel for national wildlife health programs. These difficult metrics could probably be measured with some degree of creativity and certainty. A key point identified by the group was that 
the outcomes of the program should feed back into refining and re-defining the program's mission, values, goals, and objectives and should reflect and influence the program's next steps in a process improvement and adaptive-management manner.

\section{Plenary session on additional need for a national wildlife health program}

This session allowed the group to reconvene to share additional thoughts and comments not yet discussed. Some additional thoughts were provided regarding justification for the existence of a national wildlife health program. With the current existence of national programs for public health and agriculture in most countries, it was identified that there should also be the existence of a national wildlife health program in order to complete the interconnected pieces of the One Health "puzzle". Additionally, it was discussed that because management of wildlife health is usually fragmented, a national wildlife health program allows for greater collaboration and serves as a centralized source of expertise.

Additional needs that were identified regarding wildlife health and disease included the improvement of:

- Internal and external communication within and between sectors and programs;

- Communication with other nations;

- Harmonization of case definitions;

- Standardization of scientific procedures;

- Validation of diagnostic tests; and

- Provision of knowledge and information on the importance of preventing and managing wildlife diseases.

The group discussed key points on the issue of funding for national wildlife health programs. A general consensus among the participants was that the government and policy holders should be significant contributors to the funding of a national wildlife health program because they are often the users of the information. For countries where a national wildlife health program is governmental and supports a public good, it was discussed that the government should assist in funding the program. For countries where a national wildlife health program is nongovernmental, it was discussed that funding opportunities may be outsourced. Because local and regional entities often contribute in large part to the information provided to and from a national program, however, it was recognized that coordination among local, regional, and national governmental agencies and nongovernmental organizations is key. It was stated that many outcomes and outputs are often used by the national government but these also often drive nongovernmental work. Therefore, participants agreed that funding should be shared by the national government as well as subnational governmental and nongovernmental agencies and organizations. The topic of funding a national wildlife health program stemmed from the questions of how to deal with a public good or public trust resource, what is proper management of a public good, and who is the client base. The participants recognized that close partnerships are needed among groups working with wildlife disease agencies.

\section{Plenary session on key features of a national wildlife health program}

Each participant used a flip chart to write down the three key features that a national wildlife health program should entail. In summary of these key features, the majority of participants agreed that a national wildlife health program should:

- Be a coordinated, integrated, and centralized nationalscale program for disease monitoring and surveillance, pathogen detection and discovery, and prevention and response to wildlife diseases;

- Be a reference center to provide harmonization and standardization of activities regarding wildlife health and disease;

- Provide wildlife health expertise as the primary voice to natural resource managers, the general public, and policy makers;

- Take a One Health approach to wildlife health;

- Provide efficient and trustworthy communication with the general public, natural resource managers, the media, and policy makers;

- Assist nations in meeting international standards, such as OIE and the Global Health Security Agenda; and

- Provide a capacity-building and training role to reduce disparities on local, national, and international levels.

Other key points of agreement included that a national wildlife health program should strive to be proactive, be an agent of change, and be strategic in its vision. Lastly, it was recognized that a national wildlife health program does not need to be able to deliver on all the goals or functions provided above. Rather, the group encouraged people to strategically provide services and functions within their current capacities instead of waiting for the "ideal" program. 


\section{Next steps}

\subsection{Follow-up actions}

The group listed some points of discussion for the next meeting including:

- Identifying the risks of not having a national wildlife health program;

- Identifying the benefits of having a national wildlife health program;

- Finding ways to achieve these benefits; and

- Identifying metrics of success that should be considered.

All members agreed that holding a workshop in person was invaluable. This workshop provides opportunities for wider dialogue in the future, and it was also agreed upon to continue networking and communicating among the current participants in attendance, as well as to reach out to other countries' wildlife health experts to provide more international collaboration and provide advice to new national programs in the near future. Two members were selected to put together a guidance document identifying the current ground rules and purpose of this group. A Google Group was also created, and a Google Drive to share documents. The meeting minutes were shared to the group for review.

The National Wildlife Health Programs Working Group Guidance document is provided as appendix 3 .

\section{Selected References}

Cox-Witton, K., Reiss, A., Woods, R., Grillo, V., Baker, R.T., Blyde, D.J., Boardman, W., Cutter, S., Lacasse, C., McCracken, H., Pyne, M., Smith, I., Vitali, S., Vogelnest, L., Wedd, D., Phillips, M., Bunn, C., and Post, L., 2014, Emerging infectious diseases in free-ranging wildlifeAustralian zoo based wildlife hospitals contribute to national surveillance: PLOS One, v. 9, no. 5, e95127.
Department for Environment, Food and Rural Affairs, 2009, England Wildlife Health Strategy, working together to reduce the impacts of wildlife diseases: London, $37 \mathrm{p}$.

Duff, J.P., Barlow, A., Breed, A., Duncan, D., Holmes, P., and Irvine, R.M., 2016, Wildlife disease surveillance by the Animal and Plant Health Agency: Bulletin of the Chartered Institute of Ecology and Environmental Management (CIEEM), v. 91, p. 7-10.

Duff, J.P., Holmes, J.P., and Barlow, A.M., 2010, Wildlife diseases-Surveillance turns to wildlife: Veterinary Record, v. 167 , p. $154-156$.

Kuiken, T., Ryser-Degiorgis, M.P., Gavier-Widén, D., and Gortázar, C., 2011, Establishing a European network for wildlife health surveillance: Revue Scientifique et Technique, v. 30, no. 31, p. 755-761.

Pewsner, Mirjam, Origgi, F.C., Frey, Joachim, and RyserDegiorgis, M.P., 2017, Assessing fifty years of general health surveillance of roe deer in Switzerland: A retrospective analysis of necropsy reports: PLOS One, v. 12, no. 1, accessed March 13, 2017, at http://dx.doi. org/10.1371/journal.pone.0170338.

Rijks, J.M., Saucedo, Bernardo, Spitzen-van der Sluijs, Annemarieke, Wilkie, G.S., van Asten, Alphons J.A.M., van den Broek, Jan, Boonyarittichaikij, Roschong, Stege, Marisca, van der Sterren, Fleur, Martel, An, Pasmans, Frank, Hughes, Joseph, Gröne, Andrea, van Beurden, S.J., and Kik, M.J.L., 2016, Investigation of amphibian mortality events in wildlife reveals an on-going ranavirus epidemic in the north of the Netherlands: PLOS One, v. 11, no. 6, p. 1-15.

Ryser-Degiorgis, M.P., and Segner, H., 2015, National competence center for wildlife diseases in Switzerland: Mandate, development and current strategies. Schweiz Arch Tierheilkd, v. 157 , no. 5, p. 255-266.

Tana, T., 2014, The MPI Animal General Surveillance Programme: Surveillance, v. 41, no. 2, p. 5-8. 

Appendixes 1-3 


\section{Appendix 1. List of Participants}

\begin{tabular}{|c|c|c|}
\hline Country & Name & Organization \\
\hline United States & Jonathan Sleeman & USGS National Wildlife Health Center, Madison, Wisconsin \\
\hline Great Britain & J. Paul Duff & Animal and Plant Health Agency (APHA), Penrith, Cumbria, CA11 9RR \\
\hline Sweden & Dolores Gavier-Widen & National Veterinary Institute (SVA), Uppsala, SE-75189, \\
\hline China & Hongxuan $\mathrm{He}$ & $\begin{array}{l}\text { National Research Center for Wildlife Borne Diseases, Institute of Zoology, Chinese } \\
\text { Academy of Sciences, Beijing }\end{array}$ \\
\hline $\begin{array}{l}\text { Republic of } \\
\text { Korea }\end{array}$ & Hang Lee & Seoul National University College of Veterinary Medicine, Seoul 08826 \\
\hline Netherlands & Jolianne Rijks & Dutch Wildlife Health Centre, Utrecht University, Yalelaan 1, 3584 CL Utrecht \\
\hline Switzerland & Marie-Pierre Ryser-Degiorgis & $\begin{array}{l}\text { Centre for Fish and Wildlife Health (FIWI), Vetsuisse Faculty, University of Bern, } \\
\text { Länggassstrasse 122, Postfach, } 3001 \text { Bern }\end{array}$ \\
\hline New Zealand & Toni Tana & Ministry for Primary Industries, Wellington, 6011 \\
\hline Argentina & Marcela Uhart & $\begin{array}{l}\text { Latin America Program, One Health Institute, School of Veterinary Medicine, University of } \\
\text { California, Davis }\end{array}$ \\
\hline Canada & Patrick Zimmer & Canadian Wildlife Health Cooperative, University of Saskatchewan \\
\hline
\end{tabular}




\section{Appendix 2. Workshop Program}

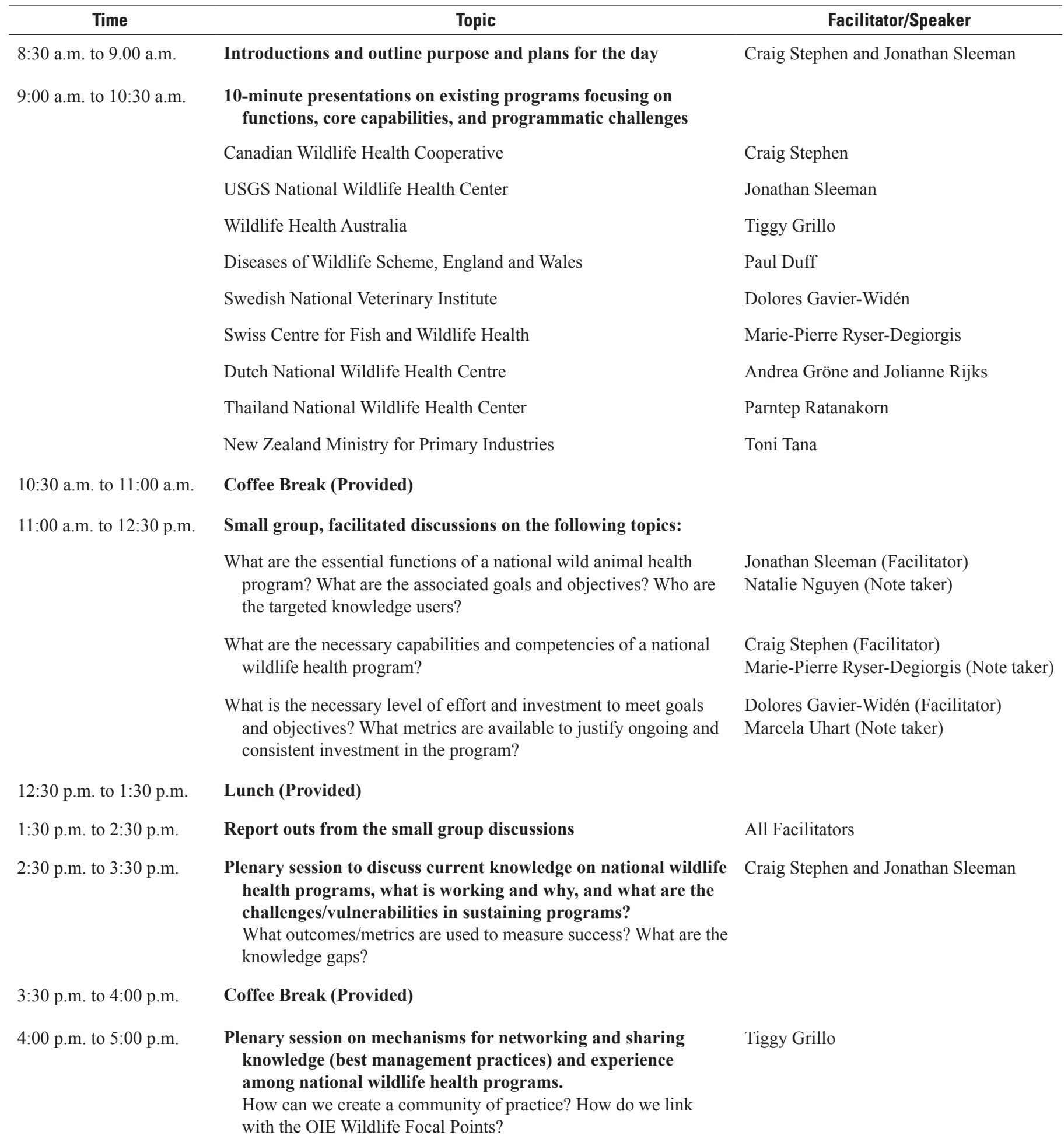




\section{Appendix 3. National Wildlife Health Programs Working Group Guidance}

\section{Purpose:}

To support a community of practice of national wildlife health programs and share experiences, insights, and evidence in order to help improve and harmonize national wildlife health programs globally.

\section{Goals:}

Facilitate open and regular exchange of information among national wildlife health groups to increase the efficiency and effectiveness of program development and delivery.

Share lessons from the development and operations of national programs with other national, sub-national, and academic networks by making outputs from the working group transparent and available to the wider wildlife health community.

\section{Background:}

First convened as a sub-group of people developing or leading national wildlife health programs, the working group met for a 1-day workshop in association with the 2016 WDA Annual Conference. The National Wildlife Health Programs working group has agreed to maintain informal connections to encourage communications and help inform assessments, planning, and policies related to wildlife health. It is anticipated that this working group will be an effective forum to discuss shared features and core competencies of national wildlife health programs.

\section{Communications:}

To aid in communications and coordination, a CWHC Google group has been formed. Individuals who are associated with this group can utilize this to communicate with the group as a whole. A contact list has been developed and is located in this Google group. Communications can be made to the group or between sub-groups and individuals.

\section{Standard of practice:}

Individuals agree that this will be an informal working group with the goal of broadening mutual understanding and approaches in the delivery of wildlife health programs through advice, expert opinion, and issue-based questions.

Discussions and postings will be treated as confidential and utilized for the purpose of furthering the above goals. Information provided within this forum will not be shared beyond group participants without the express consent of the author.

Products or outputs of the working group that are to be shared beyond the group would be made available for comment to all group members to ensure these communications are accurate and to identify the most effective means of disseminating the information.

\section{Membership:}

Members will include individuals with active involvement in developing and (or) delivering national wildlife health programs. The group is targeting people with responsibilities for designing, marketing, managing, and sustaining national wildlife health programs. All participants can suggest new members for this working group. Bimonthly conference calls will be scheduled. 



\section{这}

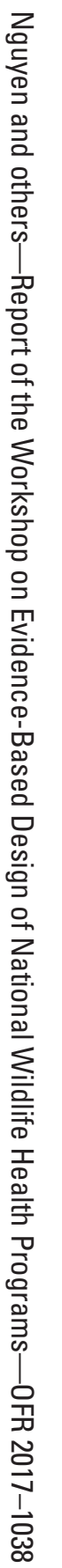

